\title{
The Impact of Blue Light Cystoscopy with Hexaminolevulinate (HAL) on Progression of Bladder Cancer - A New Analysis
}

\author{
Ashish M. Kamat ${ }^{\mathrm{a}, *}$, Michael Cookson ${ }^{\mathrm{b}}$, J. Alfred Witjes ${ }^{\mathrm{c}}$, Arnulf Stenzl ${ }^{\mathrm{d}}$ and H. Barton Grossman ${ }^{\mathrm{e}}$ \\ ${ }^{a}$ Department of Urology, University of Texas M D Anderson Cancer Center, Houston, TX, USA \\ ${ }^{\mathrm{b}}$ Department of Urology, University of Oklahoma, Oklahoma City, OK, USA \\ ${ }^{\mathrm{c}}$ Radboud University, Nijmegen Medical Centre, Nijmegen, Netherlands \\ ${ }^{\mathrm{d}}$ Department of Urology, University Hospital, Tübingen (AS), Germany \\ ${ }^{\mathrm{e}}$ Department of Urology, University of Texas M D Anderson Cancer Center, Houston, TX, USA
}

\begin{abstract}
.
Background: The International Bladder Cancer Group (IBCG) recently proposed a new definition of disease progression in non-muscle invasive bladder cancer (NMIBC), including change in T-stage, change to T2 or higher or change from low to high grade.

Objective: To establish whether blue light cystoscopy with hexaminolevulinate (HAL) impacts the rate of progression and time to progression using the revised definition.

Methods: An earlier long-term follow-up of a controlled Phase III study reported outcomes following blue light cystoscopy with HAL (255 patients) or white light (WL) cystoscopy (261 patients) in NMIBC patients. The data was re-analysed according to the new definition.

Results: In the original analysis, after 4.5 years (median), eight HAL and 16 WL patients were deemed to have progressed (transition from NMIBC to muscle invasive bladder cancer, (T2-4)).

According to the new definition, additional patients in both groups were found to have progressed: 31 (12.2\%) HAL vs 46 $(17.6 \%) \mathrm{WL}(p=0.085)$ with four $(1.6 \%) \mathrm{HAL}$ and $11(4.2 \%) \mathrm{WL}$ patients progressing from Ta to CIS. Time to progression was longer in the HAL group $(p=0.05)$.

Conclusions: Applying the new IBCG definition there was a trend towards a lower rate of progression in HAL patients, particularly in those progressing from Ta to CIS. Time to progression was significantly prolonged. This suggests that patients should receive blue light cystoscopy with HAL rather than WL at resection. Adoption of the new definition could allow more patients at risk of progression to be treated appropriately earlier.
\end{abstract}

Keywords: Hexaminolevulinate, bladder cancer, progression, NMIBC, blue light cystoscopy

\section{INTRODUCTION}

Progression is one of the most important clinical outcomes in non-muscle invasive bladder cancer (NMIBC) as it indicates a worsening of disease.

\footnotetext{
${ }^{*}$ Correspondence to: Ashish M. Kamat, Department of Urology, University of Texas M D Anderson Cancer Center, Houston, TX, USA. Tel.: +1 713792 3250; E-mail: akamat@mdan derson.org.
}

Despite this, the definition of "progression" is neither precise nor consistent and until now, there has been no standard definition which can be determined by reproducible and reliable procedures.

As a result, previous studies have employed different definitions for disease progression, or have failed to specify any method of defining advancing disease. An increase in stage is the most commonly used definition, but this fails to include other important 
indicators of advancing disease such as progression to invasion of the lamina propria or increases in grade. Prevention of progression is a major goal in the treatment of NMIBC but a standardized definition is required for comparisons to be meaningful.

A recent publication by the International Bladder Cancer Group (IBCG) highlighted this issue and the need for a new standard definition of disease progression in NMIBC to more accurately determine patient prognosis and to provide a better comparison of treatment options [1].

The new definition proposed by the IBCG includes any one of: an increase in $\mathrm{T}$ stage from Ta to CIS or T1, CIS to T1 (indicating invasion of the lamina propria), development of $\mathrm{T} 2$ or greater, lymph node disease (N+), distant metastasis (M1) or an increase in grade from low to high.

In the current analysis, the new definition of progression was applied to data from a previous longterm follow-up study of blue light cystoscopy with HAL in patients with NMIBC bladder tumors [2]. In the original analysis the definition of progression was from non-muscle invasive to muscle invasive bladder cancer. The original analysis showed that the impact of improved tumor detection and resection using blue light cystoscopy with HAL resulted in a trend in progression rates in favor of the patients undergoing blue light cystoscopy with HAL. The current re-analysis applied the new IBCG definition to see whether the progression rate would be reduced and time to progression would be prolonged using blue light cystoscopy with HAL.

\section{MATERIALS AND METHODS}

An earlier controlled, randomized, Phase III multicenter study investigated the impact of improved detection of NMIBC using blue light cystoscopy with HAL on early recurrence rates [3].
In the Phase III study, all patients underwent WL cystoscopy followed by TURB if indicated. Patients in the HAL group underwent additional inspection with HAL and inspection under blue light before and after TURB, where indicated. Follow up cystoscopies were carried out with white light at 3,6 and 9 months or until recurrence. After completion of the study patients were treated according to standard clinical practice.

An extension to the Phase III study was carried out extending the follow up to approximately 4.5 years [2]. The extension study collected data retrospectively and time from inclusion to follow-up was not pre-specified. The study extension obtained additional follow up information for 255 HAL (94\%) and $261 \mathrm{WL}(93 \%)$ patients with a median follow up of 55.1 and 53.0 months respectively.

The data from the extension study have now been re-analysed at time points of 1,3 and 4.5 years using the new definition for progression proposed by the IBCG [1].

Fischer's exact test was used to test differences in rate of progression and Kaplan Meier estimates to test differences in time to progression.

\section{RESULTS}

Using the new IBCG definition, 31 (12.2\%) HAL and $46(17.6 \%)$ WL patients had progressed by 4.5 years $(p=0.085)$, see Table 1 . This trend was particularly pronounced in patients progressing from Ta to CIS: four $(1.6 \%)$ in the HAL group compared to 11 $(4.2 \%)$ in the WL group.

The numbers progressing in year one were 15 (5.9\%) in the HAL group and $23(8.8 \%)$ in the WL group; while by year three the numbers were 26 $(10.2 \%)$ and $38(14.6 \%)$ respectively.

Time to progression was significantly longer in the HAL group $(p=0.05)$, see Fig. 1.

Table 1

Progression rate at 4.5 years (ITT recurrence population)

\begin{tabular}{|c|c|c|c|c|c|c|}
\hline & \multicolumn{2}{|c|}{1 year } & \multicolumn{2}{|c|}{3 years } & \multicolumn{2}{|c|}{4.5 years } \\
\hline & HAL $(n=255)$ & WL $(n=261)$ & HAL $(n=255)$ & WL $(n=261)$ & HAL $(n=255)$ & $\mathrm{WL}(n=261)$ \\
\hline Number (\%) of patients with progression & $15(5.9)^{ \pm}$ & $23(8.8)$ & $26(10.2)^{\dagger}$ & $38(14.6)$ & $31(12.2)^{*}$ & $46(17.6)$ \\
\hline Ta to CIS & $2(0.8)$ & $5(1.6)$ & $2(0.8)$ & $10(3.8)$ & $4(1.6)$ & $11(4.2)$ \\
\hline Ta to $\mathrm{T} 1$ & $4(1.6)$ & $7(2.7)$ & $9(3.5)$ & $8(3.0)$ & $10(3.9)$ & $9(3.4)$ \\
\hline $\mathrm{Ta}$ to $\geq \mathrm{T} 2$ & $2(0.8)$ & $3(1.2)$ & $4(1.6)$ & $5(1.9)$ & $4(1.6)$ & $8(3.1)$ \\
\hline CIS to $\mathrm{T} 1$ & $2(0.8)$ & $1(0.4)$ & $3(1.2)$ & $2(0.8)$ & $3(1.2)$ & $2(0.8)$ \\
\hline $\mathrm{T} 1$ to $\geq \mathrm{T} 2$ & $3(1.2)$ & $3(1.1)$ & $3(1.2)$ & $5(1.9)$ & $3(1.2)$ & $5(1.9)$ \\
\hline Grade 1 to Grade 3 & 0 & $2(0.8)$ & $3(1.2)$ & $5(1.9)$ & $4(1.6)$ & $7(2.7)$ \\
\hline Death due to bladder cancer & $2(0.8)$ & $2(0.8)$ & $2(0.8)$ & $3(1.1)$ & $3(1.2)$ & $4(1.5)$ \\
\hline
\end{tabular}

${ }^{ \pm} p$-value $=0.239$ (Fisher's exact test). ${ }^{\dagger} p$-value $=0.143$ (Fisher's exact test). ${ }^{*} p$-value $=0.085$ (Fisher's exact test). 


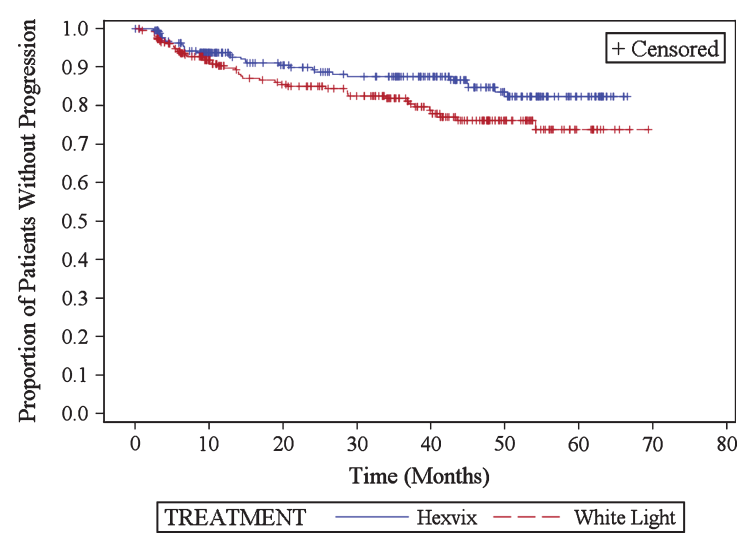

Fig. 1. Kaplan Meier plot for time to progression (ITT recurrence population).

With reference to the original analysis of the follow-up study data, there was a trend $(p=0.066)$ that fewer HAL patients progressed to muscleinvasive disease at first recurrence ( 8 vs 16 , see Table 2), a total of 24 patients. The definition of progression was transition from non-muscle invasive to muscle invasive bladder cancer, (T2-4).

Using the revised definition proposed by the IBCG, it can be seen that a number of patients in both treatment groups who were previously classified as recurring without progression had in fact progressed to a higher grade, or to CIS, a total of 77 patients (Table 2, $p=0.085$ ).

\section{DISCUSSION}

Improved detection of NMIBC tumors with HAL compared to WL cystoscopy has already been shown to lead to a lower rate of recurrence and a longer recurrence-free interval [2]. Using a new definition for disease progression in NMIBC, which aims to identify patients with high risk of developing muscle invasion at an earlier stage, we found a shorter time to progression and a trend towards a higher rate of progression in WL cystoscopy patients compared to HAL, confirming the importance of the ability of HAL to improve detection and removal of tumors.

In addition to the removal of areas of suspicious tissue, improved identification, particularly of CIS, may lead to adjustment of the treatment strategy with a subsequent impact on progression [4]. In the original study, participants with high grade bladder cancer, $\mathrm{T} 1$ or CIS were to receive an induction course of BCG followed by three weekly maintenance instillations at 3 and 6 months. Following completion of the study, patients were to be managed according to standard clinical practice.

Improved progression rates might be caused by differences in use of intravesical therapy in the period following initial treatment, as greater use of intravesical therapy could have an impact on progression. In the original study BCG was administered to 50 (19\%) participants in the HAL group and 55 (20\%) in the WL group [3]. When the study extension data is included, 123 (45\%) of the HAL group and $130(46 \%)$ of the WL group received some form of intravesical therapy [2]. As the rates of intravesical therapy were similar in the two groups, this can be ruled out as the explanation for improved progression rates in the HAL group.

The definition of progression in bladder cancer is extremely important as it indicates advancing disease. One of the most commonly used definitions of progression in NMIBC is an increase in tumor stage to muscle invasive disease (stage T2 or greater) which recognizes the fact that prognosis and treatment of patients whose tumors have invaded muscle are very different to those in patients who have NMIBC.

However, there are other indicators of advancing disease, such as invasion of the basement membrane, that signal the capability of the tumor to metastasise. These changes are rarely or inconsistently used to indicate progression.

High grade Ta lesions carry a high risk of invasion into the lamina propria and beyond, with some studies suggesting that up to $40 \%$ of patients with Ta

Table 2

Comparison of progression rates at 4.5 years (ITT recurrence population)

\begin{tabular}{|c|c|c|c|c|}
\hline & \multicolumn{2}{|c|}{$\begin{array}{l}4.5 \text { years, re-analysis } \\
\text { New definition of } \\
\text { progression }\end{array}$} & \multicolumn{2}{|c|}{$\begin{array}{c}4.5 \text { years original study } \\
\text { data Progression } \\
\text { defined as T2 - T4 }\end{array}$} \\
\hline & HAL $(n=255)$ & $\mathrm{WL}(n=261)$ & HAL $(n=255)$ & $\mathrm{WL}(n=261)$ \\
\hline Number $(\%)$ of patients with recurrence & $158(62)$ & $178(68)$ & $158(62)$ & $178(68)$ \\
\hline Number $(\%)$ recurrence without progression & $127(50)$ & $132(50.4)$ & $150(59)$ & $162(62)$ \\
\hline Number $(\%)$ recurrence with progression & $31(12.2)$ & $46(17.6)$ & $8(3)^{*}$ & $16(6)$ \\
\hline
\end{tabular}

${ }^{*} p$-value $=0.066$ (Fisher's exact test). 
tumors can experience invasion of the lamina propria, and up to $25 \%$ can experience muscle invasive disease [5-8]. As progression to T1 disease clearly indicates advancement of NMIBC the IBCG recommended that tumor changes from Ta to $\mathrm{T} 1$ be included in the definition of progression. In the original study upon which this analysis was based, there were 47 patients $(16 \%)$ in whom at least one Ta or T1 tumor was seen with blue light cystoscopy but was missed during inspection under white light [3]. In $43 \%$ of these patients the tumors that were detected with HAL only were high grade or T1 [3].

There is good evidence that grade is a better prognostic indicator of progression and mortality than recurrence [8-10] with a noticeable effect on overall survival [11]. Based on this evidence the IBCG recommended that grade progression be included in a standard definition of progression. Likewise, the development of CIS in a patient with low grade disease can be interpreted as a clear indication of grade progression [12].

The presence of CIS is of particular concern as detection rates using visual inspection are quite low. In this respect, it has been reported that detection of CIS can be improved with the addition of blue light cystoscopy to standard WL cystoscopy [3, 13-20]. In the original study, 13/41 (32\%) patients with CIS had CIS detected only with blue light and not WL $(p<0.0001)$ [3]. The significant increase in time to progression with blue light cystoscopy is clearly important and is probably due to better detection and resection at an early stage.

In the re-analysis reported here, using the new definitions, a greater number of patients at risk of progression to muscle invasive disease were identified: a total of 77 compared to 24 in the original analysis. It is clearly of great clinical importance to detect these changes at an early stage: finding changes early enables treatment decisions to be made that can minimize the risk of tumors progressing to full-scale muscle-invasion.

It is also of note that in this study more patients progressed to CIS in the WL than in the HAL group. Although the original study was not powered to show this difference, there is an indication of a trend even in this relatively small study.

In a recently published study, a retrospective analysis was carried out on 224 patients undergoing radical cystectomy (RC) for bladder cancer. It was found that patients who had previously received HAL TURB had significantly better 3 -year recurrence-free survival $(77.8 \%$ vs $52.4 \%, p=0.002)$, cancer-specific survival $(83.9 \%$ vs $59.7 \%, p=0.023)$ and overall survival (74.0\% vs $56.5 \%, p=0.037)$ following $\mathrm{RC}$ than patients who had previously undergone white light TURB [21]. Patients who had received blue light TURB (with either HAL or 5-ALA) also received a higher number of TURBs and re-resections before RC.

There have been reports of the use of HAL cystoscopy which have found no benefit in terms of progression through the use of this technique. A study by Geavlete et al. (2012) found no significant difference in progression-free survival but did not report the parameters used to define progression [22]. Also, follow up in this study was quite short at one and two years.

As was noted above, studies have variously defined progression as invasion of the detrusor muscle, progression to a higher $\mathrm{T}$ stage, or presence of CIS making comparisons difficult. We suggest that re-analysis of the data using the definition of progression proposed by the IBCG, may produce different conclusions.

Recurrence of high grade T1 disease or CIS following complete resection and effective intravesical chemotherapy is associated with an increased risk of progression that can be reduced by radical cystectomy. Therefore, it is common practice to perform cystectomy before progression to muscle invasion occurs. A more precise method for defining progression may help to avoid unnecessary cystectomies.

These findings highlight the importance of using a definition for progression that is sensitive to early changes that indicate advancing disease and a higher risk for progression. They also highlight the importance of proper detection and resection of tumors as early as possible in the disease course, the role that inspection with blue light can have in improving the detection and removal of early tumors and CIS, and the impact of this on rate of progression and time to progression.

\section{CONCLUSIONS}

Improved detection of NMIBC tumors with HAL has already been shown to affect rate of progression. A new definition by the IBCG includes indicators of advancing disease with the aim of identifying progression at an earlier stage. Applying the new definition to data from an earlier study, more patients at risk were identified in both groups, with a trend towards a lower rate of progression in HAL patients, 
particularly in those patients progressing from Ta to CIS. Also, time to progression was significantly prolonged. This suggests that patients should receive HAL rather than WL at first resection. Adoption of this broader definition could allow more patients with indicators of advancing disease, and at risk of progression to be treated appropriately at an earlier stage.

\section{SOURCE OF FUNDING}

Photocure Inc, Princeton, NJ provided funding for the statistical analysis.

\section{APPROVALS}

Approval of the study by an internal review board was not required.

\section{CLINICAL TRIAL NUMBER}

No registration was required for the re-analysis. The clinical trial number of the original study was NCT00233402 (www.clinicaltrials.govt).

\section{ACKNOWLEDGMENTS}

The authors would like to acknowledge inVentiv Health [Burlington, MA, USA] for the statistical analysis, and Jude Douglass, Healthcom Partners Ltd, Oxford, UK for writing and editorial assistance.

\section{CONFLICT OF INTEREST}

For Kamat \& Cookson: research/trial support from Photocure, NA.

\section{REFERENCES}

[1] Lamm D, Persad R, Brausi M, et al. Defining progression in nonmuscle invasive bladder cancer: It is time for a new, standard definition. J Urol 2014;191:20-7.

[2] Grossman HB, Stenzl A, Fradet Y, et al. Long-term reduction in bladder cancer recurrence with hexaminolevulinate enabled fluorescence cystoscopy. J Urol 2012;188:58-62.

[3] Stenzl A, Burger M, Fradet Y, et al. Hexaminolevulinate guided fluorescence cystoscopy reduces recurrence in patients with non-muscle invasive bladder cancer. J Urol 2010;184:1907-13.

[4] Daniltchenko D, Riedl CR, Sachs MD, et al. Longterm benefit of 5-aminolevulinic acid fluorescence assisted transurethral resection of superficial bladder cancer: 5- year results of a prospective randomized study. J Urol 2005;174:2129-33.

[5] Herr HW. Tumor progression and survival of patients with high grade, noninvasive papillary (TaG3) bladder tumors: 15-year outcome. J Urol 2000;163:60-1.

[6] Lebret T, Bohin D, Kassardjian Z, et al. Recurrence, progression and success in stage Ta grade 3 bladder tumors treated with low dose bacillus Calmette-Guerin instillations. J Urol 2000;163:63-7.

[7] van der Meijden A, Sylvester R, Collette L, Bono A, ten Kate F. The role and impact of pathology review on stage and grade assessment of stages Ta and T1 bladder tumors: A combined analysis of 5 European Organization for Research and Treatment of Cancer trials. J Urol 2000;164: 1533-7.

[8] Sylvester RJ, van der Meijden AP, Witjes JA, et al. Highgrade Ta urothelial carcinoma and carcinoma in situ of the bladder. Urology 2005;66:90-107.

[9] Millan-Rodriguez F, Chechile-Toniolo G, Salvador-Bayarri J, Palou J, Vicente-Rodriguez J. Multivariate analysis of the prognostic factors of primary superficial bladder cancer. J Urol 2000;163:73-8.

[10] May M, Brookman-Amissah S, Roigas J, et al. Prognostic accuracy of individual uropathologists in noninvasive urinary bladder carcinoma: A multicentre study comparing the 1973 and 2004 World Health Organisation classifications. Eur Urol 2010;57:850-8.

[11] Cao D, Vollmer RT, Luly J, et al. Comparison of 2004 and 1973 World Health Organization grading systems and their relationship to pathologic staging for predicting long-term prognosis in patients with urothelial carcinoma. Urology 2010;76:593-9.

[12] Chade DC, Shariat SF, Godoy G, et al. Clinical outcomes of primary bladder carcinoma in situ in a contemporary series. J Urol 2010;184:74-80.

[13] Jocham D, Witjes F, Wagner S, et al. Improved detection and treatment of bladder cancer using hexaminolevulinate imaging: A prospective, phase III multicenter study. J Urol 2005; 174:862-66.

[14] Fradet Y, Grossman HB, Gomella L, et al. A comparison of hexaminolevulinate fluorescence cystoscopy and white light cystoscopy for the detection of carcinoma in situ in patients with bladder cancer: A phase III, multicenter study. J Urol 2007;178:68-73.

[15] Jichlinski P, Guillou L, Karlsen S, et al. Hexyl aminolevulinate fluorescence cystoscopy: A new diagnostic tool for photodiagnosis of superficial bladder cancer - a multicenter study. J Urol 2003;170:226-29.

[16] Zaak D, Kriegmair M, Stepp H, et al. Endoscopic detection of transitional cell carcinoma with 5-aminolevulinic acid: Results of 1012 fluorescence endoscopies. Adult Urol 2001;57:690-4.

[17] Schmidbauer J, Witjes F, Schneller M, Donat R, Susani M, Marberger M. Improved detection of urothelial carcinoma in situ with hexaminolevulinate fluorescence cystoscopy. J Urol 2004;171:135-38.

[18] Burgués JP, Conde G, Oliva J, et al. Hexaminolevulinate photodynamic diagnosis in non-muscle invasive bladder cancer: Experience of the BLUE group. Actas Urol Esp 2011;May 26.

[19] Grossman HB, Gomella L, Fradet Y, et al. A phase III, multicenter comparison of hexaminolevulinate fluorescence cystoscopy and white light cystoscopy for the detection of superficial papillary lesions in patients with bladder cancer. J Urol 2007;178:62-7. 
[20] Burger M, Grossman HB, Droller MJ, et al. Photodynamic diagnosis of non-muscle-invasive bladder cancer with hexaminolevulinate cystoscopy: A meta-analysis of detection and recurrence based on raw data. Eur Urol 2013; http://dx.doi.org/10.1016/j.eururo.2013.03.059.

[21] Gakis G, Ngamsri T, Rausch S, et al. Fluorescence-guided bladder tumour resection: Impact on survival after radical cystectomy. World J Urol 2015;2015. doi:10.1007/s00345$015-1485-8$
[22] Geavlete B, Multescu R, Georgescu D, Jecu M, Stanescu F, Geavlete P. Treatment changes and long-term recurrence rates after hexaminolevulinate (HAL) fluorescence cystoscopy: Does it really make a difference in patients with non-muscle-invasive bladder cancer (NMIBC)? BJU Int 2012;109:549-56. 